\title{
Non-Relativistic and Relativistic Equations for the Kratzer Potential plus a Dipole in $2 D$ Systems
}

\author{
M. Heddar ${ }^{1}$ \\ M. Moumni ${ }^{1,2}$ \\ mebarek.heddar@univ-biskra.dz \\ m.moumni@univ-biskra.dz \\ M. Falek ${ }^{1}$ \\ mokhtar.Falek@univ-biskra.dz \\ ${ }^{1}$ Laboratory of Photonics and Nanomaterials (LPPNMM) \\ Department of Matter Sciences, University of Biskra, Algeria \\ ${ }^{2}$ Laboratory of Radiations and Matter (PRIMALAB) \\ Department of Physics, University of Batna, Algeria
}

May 14, 2019

\begin{abstract}
In this work, we study the wave equations in 2D Euclidian space for a new non-central potential consisting of a Kratzer term and a dipole term $V(r, \theta)=Q r^{-1}+D_{r} r^{-2}+D_{\theta} \cos (\theta) r^{-2}$. For Schrodinger equation, we obtain the analytical expressions of the energies and the wave functions of the system. For Klein-Gordon and Dirac equations, we do the study in both spin and pseudo-spin symmetries to get the eigenfunctions and the eigenvalues. We also study the dependence of energies on the parameters $D_{r}$ and $D_{\theta}$. We find that the $D_{\theta}$ term tends to dissociate the system, and thus counteracts the Coulomb binding effect, and that the $D_{r}$ term can either amplify or decrease this effect according to its sign.
\end{abstract}

\section{Contents}

1 Introduction 2

2 The Non-Central Kratzer Potential 3

3 2D Schrödinger Equation for N-C Kratzer Potential 4

3.1 Solution of Angular Equation . . . . . . . . . . . . . . . . 5

3.2 Solution of Radial Equation . . . . . . . . . . . . . . . . . . . 6

4 2D Relativistic Equations for N-C Kratzer Potential 11

4.1 Klein-Gordon Equation . . . . . . . . . . . . . . . . . 11

4.2 Dirac Equation . . . . . . . . . . . . . . . . . . . 12 
5 Solutions of the Schrödinger Type Equation 13

5.1 The Spin Symmetry Case . . . . . . . . . . . . . . . . . . . . . 13

5.2 The Pseudo-Spin Symmetry Case . . . . . . . . . . . . . . . . . . . . . . 14

6 Discussion 16

\section{Introduction}

The quantum wave equations, namely Schrödinger, Klein-Gordon and Dirac, are very good toots for the study of atomic and molecular systems. Unfortunately those equations have analytical solutions for very few type of potentials and the majority are central. The complexity of the study of multi-particle systems has forced theorists to use mean-field models and most of them are also central. These central potentials don't correspond to the reality of physical systems, except for the Coulomb potential that matches perfectly the Hydrogen because it is just a two-particle system.

When we study non central potentials, the difficulty already appears in Schrödinger equation, where the separation of variables is only possible when the potential is of the type $V(r)+V(\theta) r^{-2}+$ $V(\varphi) r^{-2} \sin ^{-2}(\theta)[1,2,3$, 4, and among them there is only few ones that can be studied analytically [5. 4]. The study of this kind of potential began with the works of Makarov [6] and Hartmann [7] in molecular systems and now they are in focus in various fields, especially in quantum chemistry and nuclear physics. For example they were used for the description of ring-shaped molecules like benzene and also for the interactions between deformed pair of nuclei [8, 9, 10, 11.

The most simple non-central potential is the electric dipole $D \cos (\theta) r^{-2}$ (or pure dipole) and it attracted attention very early because there were experimental clues that it could have bound states only if its moment exceed a critical value [12. Experiments studies followed and confirmed these results despite the fact that there is no analytical solutions for this system [13]. This potential has also attracted attention in chemistry given its applications in the anionic bounds of polar molecules [14, 15, 16, 17, and also in molecular biology in the study of electron binding mechanisms in DNA and RNA [18] (for a review see [19]).

From a theoretical point of view, the pure dipole potential has been studied in the case of 3D systems [20] and also in 1D and 2D systems 20, 21]. Theoretical studies have also focused on the potential of the non-pure dipole $Q r^{-1}+D \cos (\theta) r^{-2}$, in 3D systems [22] and in 2D ones [23]. For this last potential, both cases showed that the dipole must be below a critical value to have bound states in the system; These results are in the opposite of those of the pure dipole.

The interest for 2D systems comes from the great popularity of graphene (and co. like silicene and manganene) and also from experimental achievements with the realization of quantum gases at low dimensions [24, 25] and before that from quasi-condensate experiments [26. In 2D systems, pure dipole is present in ultrathin semiconductor layers [27, in spin-polarized atomic hydrogen absorbed on the surface of superfluid helium 28, for charged particles in a plane with perpendicular magnetic field [29] and also in gapped graphene with two charged impurities [30, 31. On the other hand, non-pure dipole potential was recently found in the case of electron pairing that stems from the spin-orbit interaction in two-dimensional quantum well [32.

In this work we consider a new type of non-central potential consisting of a combination of a Kratzer potential and a dipole term $V(r, \theta)=Q r^{-1}+D_{r} r^{-2}+D_{\theta} \cos (\theta) r^{-2}$. Our choice for this potential comes the fact that it is a better generalization of the Coulomb interaction than the non-pure dipole potential considered in [22, 23] and [32 and also for the potential richness of its applications given the number of parameters in its expression. We add a dipole term to the Kratzer 
potential to take into account the anisotropy of the distribution of charges and thus generalize the studies already made for central distributions. Our study also aims to add a new non-central system that has analytical solutions to those already presented in the literature [5, 4.

Kratzer potential was introduced to study the regularities in the band spectra of diatomic molecules [33, 34, and since then it has attracted a lot of attention because of its applications in various fields of physics and chemistry such as nuclear physics [35, molecular physics [36, quantum chemistry [37 and chemical physics [38. It is even used for the study of optical properties in semiconductor quantum dots [39]. The studies the Kratzer potential modified with angular terms followed for their possible applications in ring-shaped organic molecules [40, 41]. Recently, the Kratzer potential has been experimentally justified in 2D systems because Rydberg series of s-type excitonic states in monolayers of semiconducting transition metal dichalcogenides, which are 2D semiconductors, follow a model system of 2D Kratzer type instead of a 2D hydrogen atom 42 .

Our work will be structured as follows: after this introduction which constitutes the first section 1. we will first start by a theoretical justification of our choice of the non-central Kratzer potential in the second section 2, then we will solve the Schrödinger equation for this new potential in the case of two-dimensional systems in the third section 3 . Finally, after two sections devoted to the results of the relativistic studies [15] will come our conclusions in the sixth section 6 .

\section{The Non-Central Kratzer Potential}

We consider a system composed of a point charge $q$ in the potential of a charge distribution $Q=\int \mathrm{d} q$ (a cluster of point charges $\mathrm{d} q$ ) such as an ion and a charged particle. The extended charge $Q$ produce the following potential at the position of the test charge $q$ :

$$
V(\vec{r})=\int \frac{1}{4 \pi \varepsilon_{0}} \frac{\mathrm{d} q_{a}}{r_{a}}
$$

We choose the origin $O$ of the reference at the center of $Q$, We denote $M$ the position of $q$ and $\vec{r}$ the corresponding vector. We put $\vec{A}$ or $a$ the position of the elementary charge $\mathrm{d} q$, so the relative position of the point charge $q$ is $\vec{r}_{a}=\overrightarrow{A M}=\overrightarrow{O M}-\overrightarrow{O A}=\vec{r}-\vec{a}$; Thus we write:

$$
V(\vec{r})=\int \frac{1}{4 \pi \varepsilon_{0}} \frac{\mathrm{d} q_{a}}{\|\vec{r}-\vec{a}\|}=\int \frac{1}{4 \pi \varepsilon_{0}} \mathrm{~d} q_{a}\left[(\vec{r}-\vec{a})^{2}\right]^{-1 / 2}
$$

We use Taylor series to write the integral because we consider that the cluster's dimensions characterized by $\|\vec{a}\|$ are small compared to the whole system represented by $\|\vec{r}\|$ :

$$
V(\vec{r})=\int \frac{1}{4 \pi \varepsilon_{0}} \frac{\mathrm{d} q_{a}}{r}\left[\left(1-2 \frac{\vec{r} \cdot \vec{a}}{\vec{r}^{2}}+\frac{\vec{a}^{2}}{\vec{r}^{2}}\right)^{2}\right]^{-1 / 2}
$$

we restrict ourselves to the 1 st order of the multipole expansion:

$$
\left[\left(1-2 \frac{\vec{r} \cdot \vec{a}}{\vec{r}^{2}}+\frac{\vec{a}^{2}}{\vec{r}^{2}}\right)^{2}\right]^{-1 / 2}=1+\frac{\vec{r} \cdot \vec{a}}{r^{2}}+O\left(\frac{\vec{a}^{2}}{\vec{r}^{2}}\right)
$$


and we get:

$$
\begin{gathered}
V(\vec{r})=\frac{1}{4 \pi \varepsilon_{0}}\left(\int \frac{\mathrm{d} q_{a}}{r}+\int \frac{\mathrm{d} q_{a}}{r} \frac{\vec{r} \cdot \vec{a}}{r^{2}}\right) \\
V(\vec{r})=\frac{1}{4 \pi \varepsilon_{0}}\left(\frac{1}{r} \int \mathrm{d} q_{a}+\frac{1}{r^{2}} \int a \cos \theta_{a} \mathrm{~d} q_{a}\right)
\end{gathered}
$$

The volume element in the integral is the one around the position $\vec{a}$ of $\mathrm{d} q_{a}$, so the position $\vec{r}$ of the point charge $q$ is a constant according to this integration and the potential becomes:

$$
V(r)=\frac{1}{4 \pi \varepsilon_{0}} \frac{Q}{r}+\frac{1}{4 \pi \varepsilon_{0}} \frac{D_{r}}{r^{2}}
$$

$D_{r}$ represents the dipole moment of the cluster and it's denoted with the indice $r$ because one can consider it as a "radial" one or a dipole with moment always pointing to the test charge $q$ [4]. The relation 6 is equivalent to the Kratzer expression defined by:

$$
V_{K}(r)=d_{e}\left(\frac{r_{e}^{2}}{r^{2}}-\frac{2 r_{e}}{r}\right)
$$

where we consider that $d_{e}$ is the dissociation energy and $r_{e}$ is the equilibrium interatomic separation in the molecule [44, 45, 46].

We see that the potential is central and this may not reflect reality because the distribution is not usually perfectly symmetric. Therefore, we have to take into account the possible anisotropy in the charge distribution and to do this we consider that the positive and the negative centers of charges do not coincide in $Q$ and we denote their positions $\vec{a}_{+}$and $\vec{a}_{-}$. This two centers form an electric dipole representing this anisotropy and the potential of such a dipole is just $D_{\theta} \cos (\theta) r^{-2}$. The dipole moment $D_{\theta}$ is proportional to the distance between the two charge centers and the angle $\theta$ defines the orientation of the position $\vec{r}$ according to the dipole axis defined by $\vec{a}_{+}-\vec{a}_{-}$. We call this term the "angular" dipole to differentiate it from the "radial" one.

Adding all the terms together gives us the Coulomb potential with two dipoles and we call it a non-central (N-C) Kratzer potential:

$$
V(r, \theta)=\frac{1}{4 \pi \varepsilon_{0}} \frac{Q}{r}+\frac{1}{4 \pi \varepsilon_{0}} \frac{D_{r}}{r^{2}}+\frac{1}{4 \pi \varepsilon_{0}} \frac{D_{\theta} \cos \theta}{r^{2}}
$$

This expression takes into account the non-point character of an ionic system acting on an elementary charge and also the anisotropy in the charge distribution of this ion; It represents the first correction of the Coulomb effect of an extended charge.

\section{2D Schrödinger Equation for N-C Kratzer Potential}

We write the two-dimensional stationary Schrödinger equation for a point charge in the potential 8 and we use the polar coordinates for the Laplacian:

$$
\left[\frac{-\hbar^{2}}{2 \mu}\left(\frac{\partial^{2}}{\partial r^{2}}+\frac{1}{r} \frac{\partial}{\partial r}+\frac{1}{r^{2}} \frac{\partial^{2}}{\partial \theta^{2}}\right)+\frac{q}{4 \pi \varepsilon_{0}}\left(\frac{Q}{r}+\frac{D_{r}}{r^{2}}+\frac{D_{\theta} \cos \theta}{r^{2}}\right)\right] \psi=E \psi
$$


We put the equation in the more convenient following form:

$$
\left[\left(\frac{\partial^{2}}{\partial r^{2}}+\frac{1}{r} \frac{\partial}{\partial r}-\frac{2 \mu q Q}{4 \pi \varepsilon_{0} \hbar^{2}} \frac{1}{r}-\frac{2 \mu q D_{r}}{4 \pi \varepsilon_{0} \hbar^{2}} \frac{1}{r^{2}}\right)+\frac{1}{r^{2}}\left(\frac{\partial^{2}}{\partial \theta^{2}}-\frac{2 \mu q D_{\theta}}{4 \pi \varepsilon_{0} \hbar^{2}} \cos (\theta)\right)\right] \psi=\frac{-2 \mu E}{\hbar^{2}} \psi
$$

and we write the solution as $\psi(r, \theta)=r^{-1 / 2} R(r) \Theta(\theta)$ to get two separate equations:

$$
\begin{gathered}
\left(\frac{\partial^{2}}{\partial \theta^{2}}-E_{\theta}-\frac{2 \mu q D_{\theta}}{4 \pi \varepsilon_{0} \hbar^{2}} \cos (\theta)\right) \Theta(\theta)=0 \\
{\left[\frac{\partial^{2}}{\partial r^{2}}+\left(E_{\theta}+\frac{1}{4}-\frac{2 \mu q D_{r}}{4 \pi \varepsilon_{0} \hbar^{2}}\right) \frac{1}{r^{2}}-\frac{2 \mu q Q}{4 \pi \varepsilon_{0} \hbar^{2}} \frac{1}{r}+\frac{2 \mu E}{\hbar^{2}}\right] R(r)=0}
\end{gathered}
$$

We have to solve the angular equation 11a to find the constants $E_{\theta}$ and then we use these angular eigenvalues to solve the radial equation $11 \mathrm{~b}$, this will give us the energies $E$ of the system and also the wave functions $\psi(r, \theta)$.

\subsection{Solution of Angular Equation}

The angular equation can easily be written as a Mathieu equation [47] by defining $\theta=2 z, a=-4 E_{\theta}$ and $p=\mu q D_{\theta} /\left(\pi \varepsilon_{0} \hbar^{2}\right)$ :

$$
\frac{\partial^{2} \Theta(z)}{\partial z^{2}}+(a-2 p \cos 2 z) \Theta(z)=0
$$

The solutions of this equation are the cosine-elliptic $c e_{2 m}(z)$ and the sine-elliptic $s e_{2 m+2}(z)$ functions where $m$ is a natural number [48. The solutions of the Mathieu equation are periodic because $z$ has $\pi$ as a period and this lead us to consider the Floquet's theorem [49] or the Bloch's theorem [50. They stipulate that, for a given value of the parameter $p$, the solution is periodic only for certain values of the other parameter $a$; They are called characteristic values and denoted $a(2 m, p)$ or $a_{2 m}(p)$ for the cosine solutions and $b(2 m, p)$ or $b_{2 m}(p)$ for the sine ones.

There is no analytical expression for the Mathieu characteristic values $a_{2 m}(p)$ and $b_{2 m}(p)$, so they are usually given either numerically or graphically. This doesn't preclude that we can write approximate analytical expressions for small and large values of $p$ [51. For small values of $p$, we can express $a$ and $b$ for $m>3$ as $\left(l=4 m^{2}-1\right)$ :

$$
a_{2 m}=b_{2 m} \approx 4 m^{2}+\frac{1}{2 l} p^{2}+\frac{20 m^{2}+7}{32 l^{3}(l-3)} p^{4}+\frac{36 m^{4}+232 m^{2}+29}{64 l^{5}(l-3)(l-8)} p^{6}+\mathcal{O}\left(p^{8}\right)
$$

The coefficients of the power series of $a_{2 m}(p)$ and $b_{2 m}(p)$ are the same until the terms in $p^{2 m-2}$.

We have similar polynomials for $m \leq 3$ but with different coefficients for the $a$ 's and the $b$ 's. We note here that there is no sine solutions for $m=0$ and so there is no $b(m=0)$.

For large values of $p$, we get another polynomial $(k=2 n+1)$ :

$$
a_{n}=b_{n+1} \approx-2 p+2 k p^{1 / 2}-\frac{1}{8}\left[k^{2}+1\right]-\left[k^{3}+3 k\right] \frac{1}{2^{7} p^{1 / 2}}-\left[5 k^{4}+34 k^{2}+9\right] \frac{1}{2^{12} p}+\mathcal{O}\left(p^{-3 / 2}\right)
$$

From now on to simplify the writing, we will use the same symbol $c_{2 m}(p)$ for both the characteristic values $a_{2 m}(p)$ and $b_{2 m}(p)$. 
Using the definitions $a=-4 E_{\theta}$ and $p=\mu q D_{\theta} /\left(\pi \varepsilon_{0} \hbar^{2}\right)$ together with the relation of Mathieu parameters enable us to write the angular eigenstates as a function of the angular moment:

$$
E_{\theta}^{(2 m)}=-\frac{1}{4} c_{2 m}\left(\frac{\mu q}{\pi \varepsilon_{0} \hbar^{2}} D_{\theta}\right)
$$

From 13, we see that for small values of $D_{\theta}$ (or $p$ ), the angular solution can be put in the form:

$$
E_{\theta}^{(2 m)}=-m^{2}+P_{m}\left(D_{\theta}\right)
$$

where $P_{m}\left(D_{\theta}\right)$ is a polynomial in terms of even power of $D_{\theta}$ starting from 2. This expression will be used to validate our solutions in the limit $D_{\theta} \rightarrow 0$.

\subsection{Solution of Radial Equation}

Using the asymptotic behavior of the solutions to solve the radial equation 11b, we start with the transformation $R(r)=r^{\lambda} e^{-\beta r} f(r)$, so we get a new differential equation for $f(r)$ :

$$
\left[r \frac{d^{2}}{d r^{2}}+2(\lambda-\beta r) \frac{d}{d r}-2\left(\frac{\mu q Q}{4 \pi \varepsilon_{0} \hbar^{2}}+\lambda \beta\right)\right] f(r)=0
$$

Because parameters $\beta$ and $\lambda$ are free ones, we chose them as follows to simplify the equation:

$$
\beta^{2}=-\frac{2 \mu E}{\hbar^{2}} \& \lambda(\lambda-1)+E_{\theta}^{(m)}-\frac{2 \mu q D_{r}}{4 \pi \varepsilon_{0} \hbar^{2}}+\frac{1}{4}=0
$$

As $\psi(r, \theta)$ must be convergent, the accepted solutions for these parameters that let $R(r)$ nonsingular at $r=0$ are:

$$
\beta=\sqrt{-\frac{2 \mu E}{\hbar^{2}}} \& \lambda=\frac{1}{2}+\sqrt{-E_{\theta}^{(m)}+\frac{2 \mu q D_{r}}{4 \pi \varepsilon_{0} \hbar^{2}}}
$$

We can reduce 17 to a confluent hypergeometric type by defining a new variable $z=2 \beta r$ :

$$
\left[z \frac{d^{2}}{d z^{2}}+(2 \lambda-z) \frac{d}{d z}-\left(\frac{\mu q Q}{4 \pi \varepsilon_{0} \hbar^{2}} \frac{1}{\beta}+\lambda\right)\right] f(z)=0
$$

The solution here is just the confluent hypergeometric function:

$$
f(z)=N_{1} F_{1}\left(\lambda+\frac{\mu q Q}{4 \pi \varepsilon_{0} \hbar^{2}} \beta^{-1}, 2 \lambda, 2 \beta r\right)
$$

and the wave function of the system follows:

$$
\psi(r, \theta)=N r^{\lambda-\frac{1}{2}} e^{-\beta r} \Theta(\theta)_{1} F_{1}\left(\lambda+\frac{\mu q Q}{4 \pi \varepsilon_{0} \hbar^{2}} \beta^{-1}, 2 \lambda, 2 \beta r\right)
$$

We compute the normalization constant $N$ from the condition $\int|\psi(r, \theta)|^{2} r d r d \theta=1$ and we recall that Mathieu functions are normalized by definition to $\pi$ [48]:

$$
N=\frac{2^{\lambda} \beta^{\lambda+\frac{1}{2}}}{(2 \lambda-1) ! \pi}\left(\frac{\left(n_{r}+2 \lambda-1\right) !}{n_{r} !\left(n_{r}+2 \lambda\right) !}\right)^{1 / 2}
$$


Here we have used Laguerre polynomials of degree $n_{r}$ [48]:

$$
L_{n_{r}}^{2 \lambda-1}(2 \beta r)=\frac{\left(n_{r}+2 \lambda-1\right) !}{n_{r} !(2 \lambda-1) !}{ }_{1} F_{1}\left(-n_{r}, 2 \lambda, 2 \beta r\right)
$$

and the identity [48:

$$
\int_{0}^{\infty} e^{-q} q^{k+1}\left[L_{n}^{k}(q)\right]^{2} d q=\frac{(n+k) !}{n !}(2 n+k+1)
$$

One can also use the Appell's double series $F_{2}$ to get the same normalized constant [52].

From the asymptotic behavior of the confluent series $\left(r \rightarrow \infty \Longrightarrow{ }_{1} F_{1}=0\right)$ which leads to $\psi \rightarrow 0$ for $r \rightarrow \infty$, we find the condition of quantization:

$$
\lambda+\frac{\mu q Q}{4 \pi \varepsilon_{0} \hbar^{2}} \beta^{-1}=-n_{r}, n_{r}=0,1,2, \ldots
$$

and we use this condition with the relation 19 to obtain the spectrum of the discrete energy levels of our system:

$$
E_{n_{r}, m}=-\left[\left(\frac{4 \pi \varepsilon_{0} \hbar^{2}}{2 \mu q Q} \sqrt{\frac{\hbar^{2}}{2 \mu}}\right)\left(n_{r}+\frac{1}{2}+\sqrt{-E_{\theta}^{(m)}+\frac{2 \mu q D_{r}}{4 \pi \varepsilon_{0} \hbar^{2}}}\right)\right]^{-2}
$$

Starting from this expression, we can get the solutions of the usual 2D Kratzer potential [53, 54 . by taking the limit $D_{\theta} \rightarrow 0$, so $P_{m}\left(D_{\theta}\right) \rightarrow 0$ which lead to $E_{\theta}^{(m)}=-m^{2}$ from 16

$$
E_{n_{r}, m}^{(\text {Kratzer })}=-\left[\left(\frac{4 \pi \varepsilon_{0} \hbar^{2}}{2 \mu q Q} \sqrt{\frac{\hbar^{2}}{2 \mu}}\right)\left(n_{r}+\frac{1}{2}+\sqrt{m^{2}+\frac{2 \mu q D_{r}}{4 \pi \varepsilon_{0} \hbar^{2}}}\right)\right]^{-2}
$$

If we add the limit $D_{r} \rightarrow 0$, we find the 2D Coulomb solutions [55, 56]:

$$
E_{n_{r}, m}^{(\text {Coulomb })}=-\left(\frac{4 \pi \varepsilon_{0} \hbar^{2}}{2 \mu q Q} \sqrt{\frac{\hbar^{2}}{2 \mu}}\right)\left(n_{r}+|m|+\frac{1}{2}\right)^{-2}
$$

Comparing with these results, we use the notations of the 2 D hydrogen atom $n=n_{r}+|m|$ and we write the energy eigenvalues of the system $\left(E_{\theta}^{(m)}\right.$ is replaced from 15):

$$
E_{n, m}=-\left[\left(\frac{4 \pi \varepsilon_{0} \hbar^{2}}{\mu q Q} \sqrt{\frac{\hbar^{2}}{2 \mu}}\right)\left(n-|m|+\frac{1}{2}+\sqrt{\frac{1}{4} c_{2 m}\left(\frac{\mu q}{\pi \varepsilon_{0} \hbar^{2}} D_{\theta}\right)+\frac{2 \mu q D_{r}}{4 \pi \varepsilon_{0} \hbar^{2}}}\right)\right]^{-2}
$$

For our numerical computations, we use the same considerations as those of molecular systems. So we choose the extended charge as a positive ion and the point charge is an electron, and we get two opposite charges equal in magnitude $q=-Q=-e$. We use the Hartree atomic units where $\hbar=e=\mu=4 \pi \varepsilon_{0}=1$ and the energies become:

$$
E_{n, m}=-2\left(n-|m|+\frac{1}{2}+\sqrt{\frac{1}{4} c_{2 m}\left(4 D_{\theta}\right)+2 D_{r}}\right)^{-2}
$$




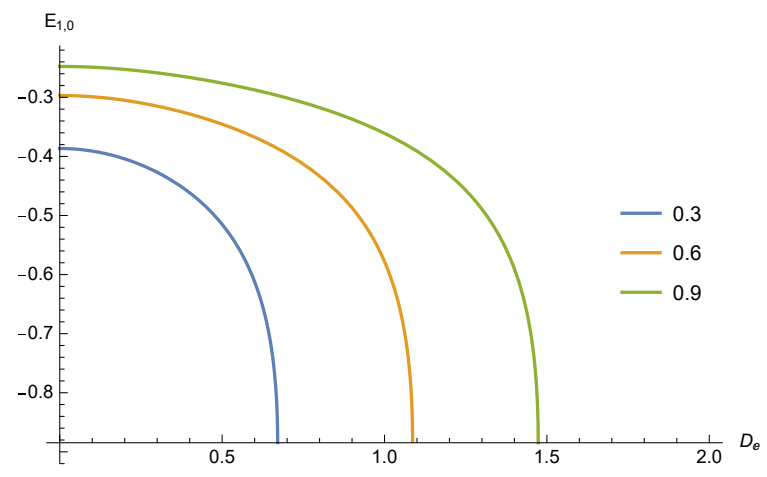

Figure 1: $E(1,0)$ vs $D_{\theta}$ for $D_{r}=0.3,0.6$ and 0.9

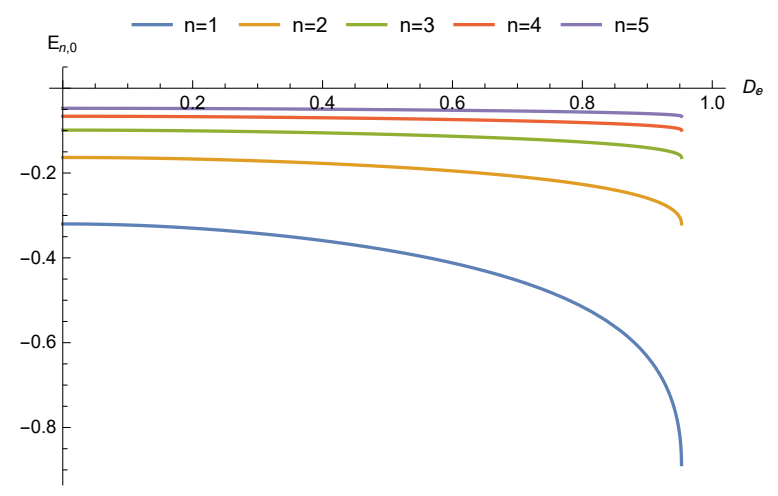

Figure 2: $E(n, 0)$ vs $D_{\theta}$ for $D_{r}=0.5$ and $n=1,2,3,4$ and 5

We note in this relation of the energies, that the angular dipole removes the degeneracy of the sine and cosine states for $m \neq 0$. This degeneracy is restored when the angular moment vanishes since the two Mathieu's characteristic parameters $a_{2 m}$ and $b_{2 m}$ have the same limit in this case 13 . The result restores those of the ordinary Kratzer potential (or Coulomb potential) where the wave function of each level $E_{n, m}$ is a linear combination of both sine and cosine states. For the $s$-states $(m=0)$, we only find the cosine solutions because the sine solutions are absent in this case.

Through the expression 31, we see that the behavior of the energies follows essentially that of the Mathieu's parameters and thus the angular moment, whereas the effect of the radial moment merely shifts the energies to larger or smaller values according to its sign. The sign of the angular moment doesn't affect the results because the parameters $c_{2 m}$ are even functions. Of course, the energies increase with the $n$ and decrease with the $m$ but the main effect of the $m$ is to extend the allowed region for the values of the angular momentum. We also note that the energies corresponding to the $c e_{2 m}(z)$ solutions are larger than the $s e_{2 m}(z)$ ones and this is caused by the fact that the $a_{2 m}$ are bigger than the $b_{2 m}$. (see figures 1 [1] 1 国 were dashed lines are for sine solutions).

The main remark that can be drawn from 31 is that there is an essential condition for the system to have bound states:

$$
\frac{1}{4} c_{2 m}\left(4 D_{\theta}\right)+2 D_{r}>0
$$




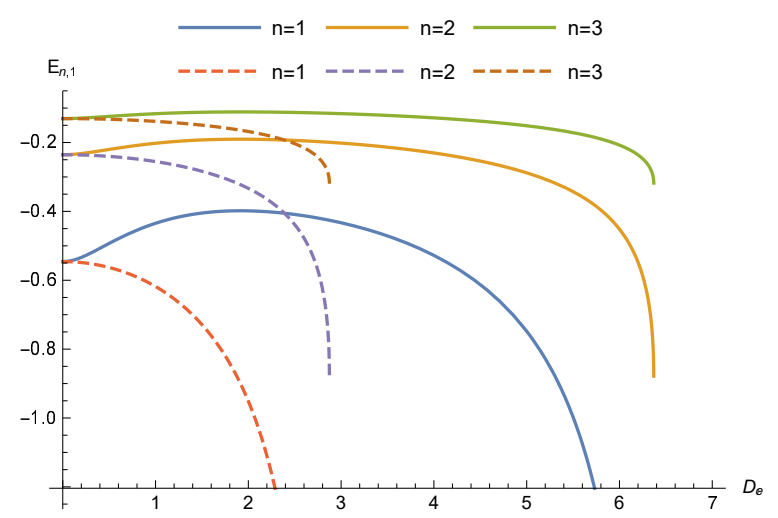

Figure 3: $E(n, 1)$ vs $D_{\theta}$ for $D_{r}=0.5$ and $n=1,2,3$

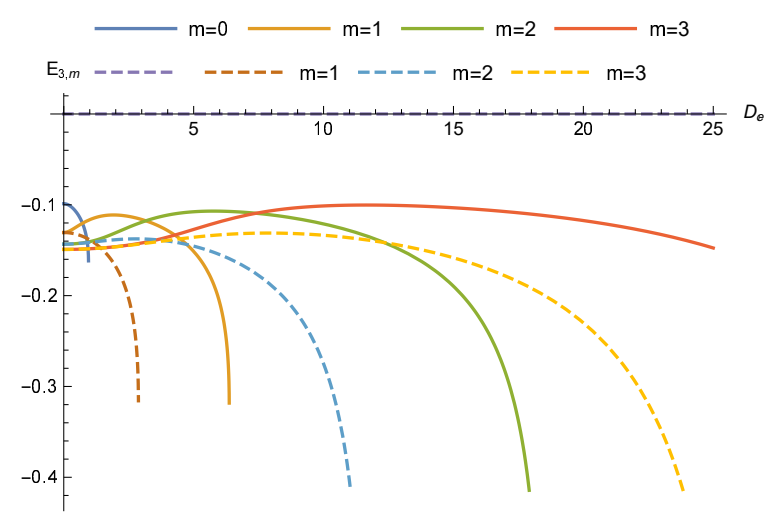

Figure 4: $E(3, m)$ vs $D_{\theta}$ for $D_{r}=0.5$ and $m=0,1,2$ and 3 


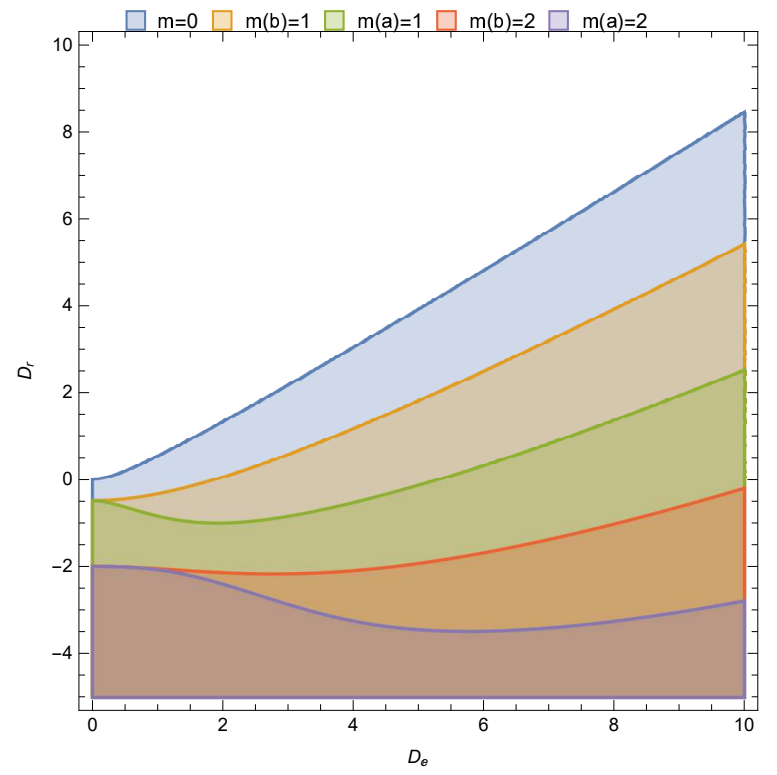

Figure 5: Forbiden regions of $D_{r}$ and $D_{\theta}$ for $m=0,1,2$

$\begin{array}{ccccc}D_{r} \backslash m & 0 & 1 & 2 & 3 \\ -0.3 & - & 1.925 & 8.004 & 17.462 \\ +0.0 & - & 2.662 & 8.679 & 18.132 \\ +0.3 & 0.543 & 3.284 & 9.323 & 18.782 \\ +0.6 & 0.923 & 3.851 & 9.942 & 19.420 \\ +0.9 & 1.284 & 4.385 & 10.543 & 20.046\end{array}$

Table 1: Critical values for $D_{\theta}$

This condition shows that there are critical values for the two dipole moments, depending only on the quantum number $m$, that make the corresponding bound state no longer exists. If we put $D_{r}=0$, all the $s$-states $(m=0)$ are absent because the critical value for $D_{\theta}$ here is zero. We say here that the presence of radial dipole is essential for $s$-states to exist, otherwise the angular moment make them disappear. The same observation is made concerning the other $m$-states $(m>0)$, but the critical value of the angular moment is positive in all these cases and these critical values increase with $m$ and also with the values of $D_{r}$ (fig 5 ). This critical value is smaller for the sine states and this causes the spread of the spectrum of these states to be less than that of the cosine states on the axis of the angular momentum (figures 4 and 5 where the indice a is for cosine solutions and the $\mathrm{b}$ for sine ones). So the radial dipole has two effects, it moves the energies to higher values while enlarging the region of possible values of angular moment (fig 6 ).

The table 1 shows the critical values of $D_{\theta}$ for different values of $D_{r}$ and $m$ when we consider cosine solutions. 


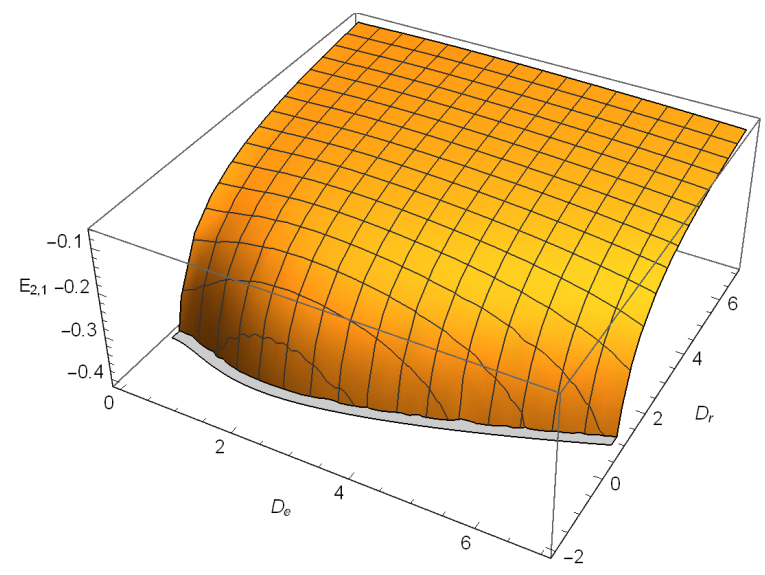

Figure 6: $E(2,1)$ vs $D_{r}$ and $D_{\theta}$

\section{2D Relativistic Equations for N-C Kratzer Potential}

We start by considering the general case of the non-central potential $V(r, \theta)=V(r)+V(\theta) / r^{2}$ in $2 \mathrm{D}$ systems. There are no analytical solutions for the Klein-Gordon and Dirac equations for this potentials, but both equations reduce to the same Schrödinger type equation if we consider the cases of spin and pseudo-spin symmetries. These symmetries have been considered for the first time in shell models of nuclear physics, where protons and neutrons are treated in the same way [57, 58. Then they became essential symmetries in relativistic theories [59, 60] and since then their studies have increased considerably in these systems 61. These symmetries were also used to study the relativistic theory of both central and ring-shaped Kratzer potentials 62, 63.

We will consider the Klein-Gordon equation first then the Dirac equation and we start with the spin symmetry case and then the pseudo-spin one.

\subsection{Klein-Gordon Equation}

The stationary Klein-Gordon equation for a single charge $q$ in both scalar $S(\vec{r})$ and vector $U(\vec{r})$ potentials is written as:

$$
\left[c^{2} p^{2}-(E-U(\vec{r}))^{2}+\left(\mu c^{2}+S(\vec{r})\right)^{2}\right] \psi(\vec{r})=0
$$

The denominations vector potential is used for the energy $U$ but it isn't vectorial in any way. The vectorial potential $\vec{A}(\vec{r})$ comes with the momentum $\vec{p}$ and is zero in our case.

Spin or pseudo-spin symmetry are defined by $S(\vec{r})= \pm U(\vec{r})$ and the two reduce the wave equation 33 to the following second order equation:

$$
\left[c^{2} p^{2}+2\left(E \pm \mu c^{2}\right) U(\vec{r})-\left(E^{2}-\mu^{2} c^{4}\right)\right] \psi(\vec{r})=0
$$

The equation is easily written as a Schrödinger equation with the transformations:

$$
\left(\frac{E}{\mu c^{2}} \pm 1\right) U(\vec{r}) \longrightarrow U(\vec{r}) \& \frac{1}{2}\left(\frac{E^{2}}{\mu c^{2}}-\mu c^{2}\right) \longrightarrow E
$$


Here we get a system where the potential depends on the energy. These energy dependant potentials have been considered for a long time when the relativistic effects began to be taken into account in quantum physics 64, 65, 66. Recently a lot of works were devoted to this type of potentials 67, 68, 69] (and the references therein).

\subsection{Dirac Equation}

We consider now the stationary Dirac equation:

$$
\left[c \vec{\alpha} \cdot \vec{p}+\beta\left(\mu c^{2}+S(\vec{r})\right)-(E-U(\vec{r}))\right] \psi(\vec{r})=0
$$

and we use the Pauli-Dirac representation:

$$
\vec{p}=-i \hbar \vec{\nabla} ; \vec{\alpha}=\left(\begin{array}{cc}
0 & \vec{\sigma} \\
\vec{\sigma} & 0
\end{array}\right) ; \beta=\left(\begin{array}{cc}
I & 0 \\
0 & -I
\end{array}\right)
$$

where $\vec{\sigma}$ is the vector of Pauli matrices and $I$ is the $2 \times 2$ identity matrix.

We write the wave function as a two component vector of the Pauli-Dirac representation:

$$
\psi(\vec{r})=\left(\begin{array}{l}
\varphi(\vec{r}) \\
\chi(\vec{r})
\end{array}\right)
$$

and we obtain two coupled differential equations:

$$
\begin{aligned}
& c \vec{\alpha} \cdot \vec{p} \chi(\vec{r})=\left[E-U(\vec{r})-\mu c^{2}-S(\vec{r})\right] \varphi(\vec{r}) \\
& c \vec{\alpha} \cdot \vec{p} \varphi(\vec{r})=\left[E-U(\vec{r})+\mu c^{2}+S(\vec{r})\right] \chi(\vec{r})
\end{aligned}
$$

If we consider spin symmetry, where $S(\vec{r})=U(\vec{r})$, the system is decoupled by writing:

$$
\chi(\vec{r})=\frac{c \vec{\alpha} \cdot \vec{p}}{E+\mu c^{2}} \varphi(\vec{r})
$$

and we get the following second order equation:

$$
\left[c^{2} p^{2}+2\left(E+\mu c^{2}\right) U(\vec{r})-\left(E^{2}-\mu^{2} c^{4}\right)\right] \varphi(\vec{r})=0
$$

In the same way, using pseudo-spin symmetry relation $S(\vec{r})=-U(\vec{r})$, we write:

$$
\varphi(\vec{r})=\frac{c \vec{\alpha} \cdot \vec{p}}{E-\mu c^{2}} \chi(\vec{r})
$$

The system is then decoupled and we get the following second order equation:

$$
\left[c^{2} p^{2}+2\left(E-\mu c^{2}\right) U(\vec{r})-\left(E^{2}-\mu^{2} c^{4}\right)\right] \chi(\vec{r})=0
$$

We see that the two equations 39 and 41 are equivalent to the equations 34

We start by studying the spin-symmetry case and then we deduce the solutions for pseudo-spin symmetry by using the transformation $E \rightarrow-E$ (and we add $\chi \leftrightarrows \varphi$ for Dirac equation). 


\section{Solutions of the Schrödinger Type Equation}

\subsection{The Spin Symmetry Case}

The Schrödinger type equation for the spin-symmetry case is 39

$$
\left[c^{2} p^{2}+2\left(E+\mu c^{2}\right) U(\vec{r})-\left(E^{2}-\mu^{2} c^{4}\right)\right] \psi(\vec{r})=0
$$

with the potential energy:

$$
U(r, \theta)=e V(r, \theta)=-Z \frac{e^{2}}{r}+e \frac{D_{r}}{r^{2}}+e \frac{D_{\theta} \cos \theta}{r^{2}}
$$

We use the polar coordinates and the same transformation as before $\psi(r, \theta)=r^{-1 / 2} R(r) \Theta(\theta)$ to get two separate equations:

$$
\begin{gathered}
\left(\frac{\mathrm{d}^{2}}{\mathrm{~d} \theta^{2}}-2 \frac{E+\mu c^{2}}{\hbar^{2} c^{2}} e D \cos \theta\right) \Theta(\theta)=E_{\theta} \Theta(\theta) \\
{\left[\frac{\mathrm{d}^{2}}{\mathrm{~d} r^{2}}+\left(E_{\theta}-2 \frac{E+\mu c^{2}}{\hbar^{2} c^{2}} e D_{r}+\frac{1}{4}\right) \frac{1}{r^{2}}+2 \frac{E+\mu c^{2}}{\hbar^{2} c^{2}} Z e^{2} \frac{1}{r}\right] R(r)=-\frac{E^{2}-\mu^{2} c^{4}}{\hbar^{2} c^{2}} R(r)}
\end{gathered}
$$

These equations are equivalent to those of the non-relativistic case $11 \mathrm{a}$ and $11 \mathrm{~b}$ but we have to consider the shifts 35. So we use the same steps to solve 46a and 46b and we get two new eigenvalues relations coming from both angular and radial equations:

$$
\begin{gathered}
E_{\theta}^{(2 m)}=-\frac{1}{4} c_{2 m}\left(4 \frac{E_{n, m}+2 \mu c^{2}}{\hbar^{2} c^{2}} e D_{\theta}\right) \\
E_{\theta}^{(2 m)}=2 \frac{E_{n, m}+2 \mu c^{2}}{\hbar^{2} c^{2}} e D_{r}-\left(n-|m|+\frac{1}{2}-Z \alpha \frac{E_{n, m}+2 \mu c^{2}}{\sqrt{\mu^{2} c^{4}-\left(E_{n, m}+\mu c^{2}\right)^{2}}}\right)^{2}
\end{gathered}
$$

We used the non-relativistic energies $E-\mu c^{2}$ and we denoted them $E_{n_{r}, m}$ or $E_{n, m}$ where $n=n_{r}+|m|$ ( $\alpha$ is the fine structure constant).

For the wavefunctions we get the same relation as 22 but with the new parameters $\beta$ and $\lambda$ :

$$
\beta^{2}=-\frac{E^{2}-\mu^{2} c^{4}}{\hbar^{2} c^{2}} \& \lambda=\frac{1}{2}+\sqrt{-E_{\theta}^{(2 m)}+2 \frac{E+\mu c^{2}}{\hbar^{2} c^{2}} e D_{r}}
$$

The same relations are used in the expression of the normalization constant 23 .

The non-relativistic limit is obtained by neglecting the term $E_{n, m}$ beside the factor $2 \mu c^{2}$ in $47 \mathrm{a}$, then we replace in $47 \mathrm{~b}$ and we use the Taylor series according to $\alpha^{2}$ :

$$
E_{n, m}=-\frac{8 \mu c^{2} Z \alpha^{2}}{\left(2 n_{r}+2 \sqrt{-E_{\theta}^{(2 m)}+4 \frac{\mu \alpha c}{e \hbar} D_{r}}+1\right)^{2}}+\frac{32 \mu c^{2} Z^{2} \alpha^{4}}{\left(2 n_{r}+2 \sqrt{-E_{\theta}^{(2 m)}+4 \frac{\mu \alpha c}{e \hbar} D_{r}}+1\right)^{4}}+O\left(\alpha^{6}\right)
$$

This result generalizes the one found in the non-relativistic case 31 and the ones found for non-relativistic non-pure dipole by putting $D_{r}=0$ [23]. One can also found the energies of the 
relativistic Kratzer potential using $D_{\theta}=0$ or $E_{\theta}^{(2 m)}=-m^{2}$ [71]. The two conditions $D_{r}=0$ and $D_{\theta}=0$ together give the energies of the Coulomb potential [72, 2].

We use the Hartree units $\left(\mu=e=4 \pi \epsilon_{0}=\hbar=1\right.$ and $\left.c=1 / \alpha\right)$ for the numerical computations and we apply for $Z=1$ :

$$
\begin{gathered}
E_{\theta}^{(2 m)}=-\frac{1}{4} c_{2 m}\left(4\left(E_{n, m} \alpha^{2}+2\right) D_{\theta}\right) \\
E_{\theta}^{(2 m)}=2\left(E_{n, m} \alpha^{2}+2\right) D_{r}-\left(n-|m|+\frac{1}{2}-Z \alpha \frac{E_{n, m} \alpha^{2}+2}{\sqrt{1-\left(E_{n, m} \alpha^{2}+1\right)^{2}}}\right)^{2}
\end{gathered}
$$

And the non-relativistic limit becomes:

$$
E_{n, m}=-\frac{2}{\left(n_{r}+\frac{1}{2}+\sqrt{\frac{1}{4} c_{2 m}\left(8 D_{\theta}\right)+4 D_{r}}\right)^{2}}+\frac{8 \alpha^{2}}{\left(n_{r}+\frac{1}{2}+\sqrt{\frac{1}{4} c_{2 m}\left(8 D_{\theta}\right)+4 D_{r}}\right)^{4}}+O\left(\alpha^{6}\right)
$$

We see here that 51 differs from 31 by a factor of 2 in front of the dipoles moments $D_{\theta}$ and $D_{r}$. This factor comes from the addition of scalar and vector potentials in spin-symmetry case which gives a Schrödinger equation with a potential $2 V$ instead of $V$ in ordinary theory [73, 74].

We cannot solve the system of equations $50 \mathrm{a}$ and $50 \mathrm{~b}$ analytically because Mathieu characteristics don't have inverse functions. Nevertheless, this system can be solved using graphical methods by seeking the intersection points of the graphs representing the two equations.

Equation 50b shows that $E_{\theta}$ has an inverted and non-symmetric parabolic shape and the intersection point with the plots representing 50a can not exceed its maximum; This limitation gives the critical dipole moments for each quantum numbers. Unlike the non-relativistic case where $D_{\text {crit }}$ depends only of the value of $m$, its values are here weakly dependent on the other quantum number $n$. This dependence on $n$ comes from the presence of the energies $E_{n, m}$ with $D$ in the angular eigenvalues 50a and these energies depend on $n$ as can be seen from 50b. The weakness of this dependence comes from the presence of the factor $\alpha^{2}$ with $E_{n, m}$.

The study of the dependence of the energies according to the values of $D_{\theta}$ shows that this moment increases the energies of the system to a maximum value and then its effect is transformed into a decrease thereof; This shape follows that of the $c_{2 m}$ and it is common to all levels but decreases with increasing $n$. The effect of $D_{r}$ can be summarized in a shift of the energies to larger or smaller values depending on its sign (Figures 7 and 8 ).

We mention here that the non-relativistic approximation 51 can be used as a quasi-analytical solution since it gives results in excellent agreement with those computed numerically (fig9).

\subsection{The Pseudo-Spin Symmetry Case}

The Schrödinger type equation for the pseudo-spin-symmetry case is 43

$$
\left[c^{2} p^{2}+2\left(E-M c^{2}\right) U(\vec{r})-\left(E^{2}-M^{2} c^{4}\right)\right] \psi(\vec{r})=0
$$

Following the same procedure as that of the spin case, we end up with two relations that come from the eigenvalues of radial and angular equations. We find the following relations for the non- 


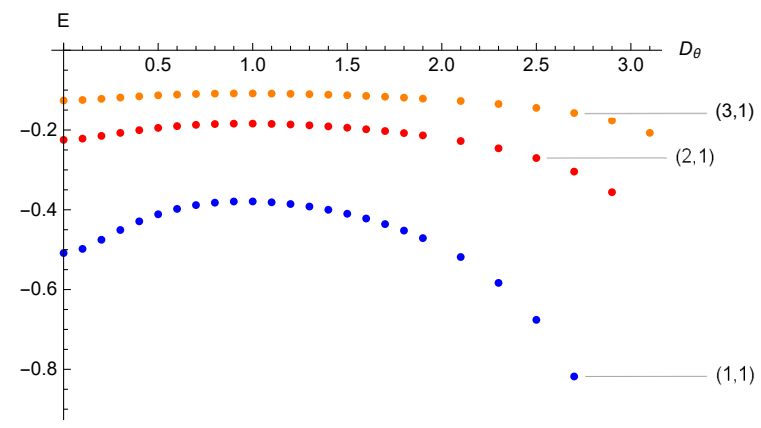

Figure 7: Relativistic $E(n, 1)$ vs $D_{\theta}$ for $D_{r}=0.3$ and $n=1,2$ and 3

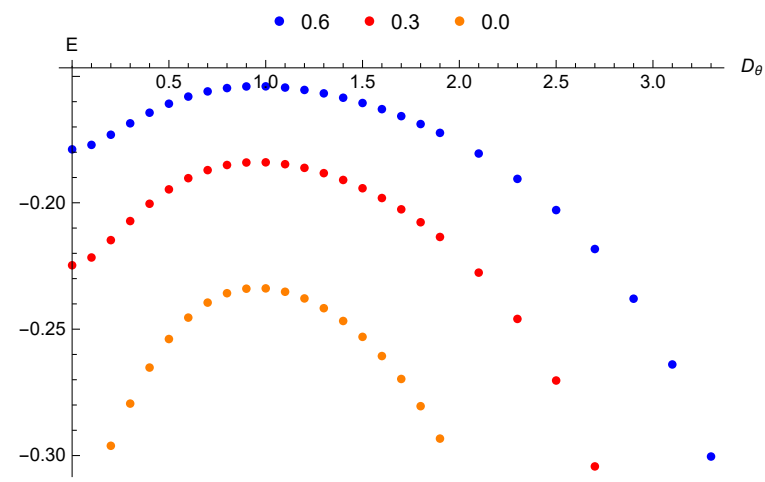

Figure 8: $E(2,1)$ vs $D_{\theta}$ for $D_{r}=0,0.3$ and 0.6

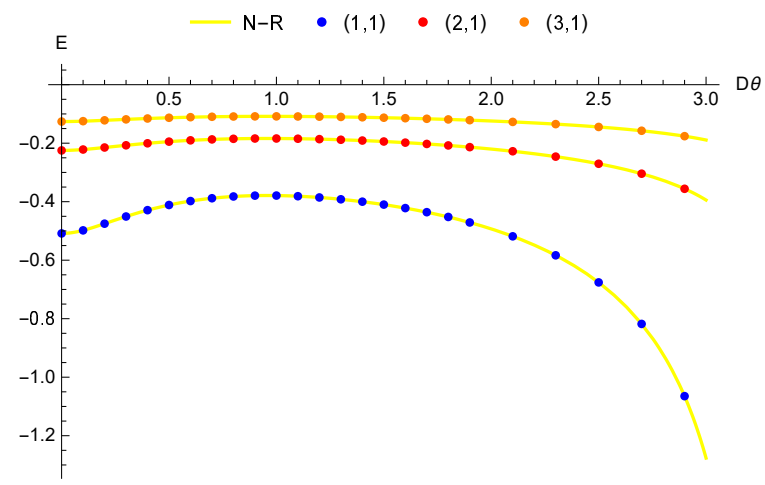

Figure 9: Relativistic and Non-Relativistic $E(1,1), E(2,1)$ and $E(3,1)$ vs $D_{\theta}$ for $D_{r}=0.3$ 
relativistic energies of the system $E_{n, m}=E-\mu c^{2}$ in Hartree units:

$$
\begin{gathered}
E_{\theta}^{(2 m)}=-\frac{1}{4} c_{2 m}\left(4 E_{n, m} \alpha^{2} D\right) \\
E_{\theta}^{(2 m)}=2 E_{n, m} \alpha^{2} D_{r}-\left(n-|m|+\frac{1}{2}-Z \alpha \frac{E_{n, m} \alpha^{2}}{\sqrt{1-\left(E_{n, m} \alpha^{2}+1\right)^{2}}}\right)^{2}
\end{gathered}
$$

The main difference between these equations and those of the spin symmetry case (50a, 50b) is the absence of factor 2 in front of the $\alpha^{2}$ term. This means that the graphs representing the radial solution $53 \mathrm{~b}$ are almost linear and that the parameter $p$ inside the Mathieu characteristics 53a is very small. Our calculations show that we have to consider very large radial moments $\left(D_{r}>100 a . u\right.$. $)$ to find solutions higher than $-200 a . u$. These results are outside the regions of interest for the energies of the atomic systems and support those of works that consider only the case of spin symmetry in their studies [71, 74, 75].

\section{Discussion}

In this work, we studied the potential $V(r, \theta)=Q r^{-1}+D_{r} r^{-2}+D_{\theta} \cos (\theta) r^{-2}$ for $2 D$ quantum systems in both non-relativistic and relativistic cases. We solved the Schrödinger equation analytically and studied the relativistic spectrum for Klein-Gordon and Dirac equations in both spin and pseudo-spin symmetry cases.

In the non-relativistic case, the spectrum shows that the energies follow mainly the behavior of Mathieu's characteristic parameters and thus the angular moment $D_{\theta}$, whereas the effect of the radial moment $D_{r}$ is merely a shift in these energies to larger or smaller values according to its sign. We have showed also that there is an essential condition for bound states to exist, which is: $c_{2 m}\left(4 D_{\theta}\right)+8 D_{r}>0$. This condition imposes a critical value for the angular moment $D_{\theta}$, depending on the value of $m$, otherwise the corresponding bound state disappears. These critical values of $D_{\theta}$ depend also on the value of $D_{r}$ and the negative value of this moment which makes $c_{2 m}\left(4 D_{\theta}\right)+8 D_{r}=0$ is also a critical value for the radial moment. So we see that by increasing, the radial dipole displaces the energies towards the larger values while widening the region of the possible values of the angular moment.

In the relativistic cases the eigenfunctions are determined analytically but the energies can only be calculated using graphical methods. Only the spin symmetry has given results corresponding to atomic systems. The behavior of the energies is the same as that of the Schrödinger spectrum but it is shifted because the Schrödinger type equation of the relativistic systems has $2 \mathrm{~V}$ as a potential instead of the potential $V$ in the ordinary Schrödinger equation. We also note that the critical values of the dipole moments $D_{r}$ and $D_{\theta}$ depend on the two quantum numbers $n$ and $m$ in the relativistic case instead of just $m$ in the case of non-relativistic systems.

We have found that the angular term removes the degeneracy found in the $\exp (i m \theta)$ part of the solutions for central potentials. This is equivalent to the effect of a constant magnetic field in $3 D$ systems, where its action removes the degeneracy of the $\exp (\operatorname{im} \varphi)$ solutions too. In both cases, the privileged direction of the interaction (dipole axis in $2 D$ and field direction in $3 D$ ) removes the degeneracy that existed due to the isotropy of the action before. 


\section{Acknowledgement}

The authors would like to thank Pr Y. Delenda for his help in writing this work and also Dr. Y. Gindikin for his comment which has enriched it.

\section{References}

[1] Khare A. and Bhaduri R.K.; Supersymmetry, Shape Invariance and Exactly Solvable Noncentral Potentials, Am. J. Phys. 62, 1008-1014 (1994)

[2] Arda A. and Sever R.; Non-central potentials, exact solutions and Laplace transform approach, J. Math. Chem. 50, 1484-1494 (2012)

[3] Alhaidari A.D. and Bahlouli H.; Electric dipole and quadrupole contributions to valence electron binding in a charge-screening environment, Eur. Phys. J. D 73, 70 (2019)

[4] Kumari N., Yadav R.K., Khare A. and Mandal B.P.; A class of exactly solvable rationally extended non-central potentials in two and three dimensions, J. Math. Phys. 59, 062103 (2018)

[5] Hautot A.; Exact motion in noncentral electric fields, J. Math. Phys. 14, 1320 (1973)

[6] Makarov A.A., Smorodinsky J.A., Valiev Kh. and Winternitz P.; A systematic search for nonrelativistic systems with dynamical symmetries, Nuovo Cimento A 52, 1061 (1967)

[7] Hartmann H.; Die Bewegung eines Körpers in einem ringförmigen Potentialfeld, Theor. Chim. Acta 24, 201 (1972)

[8] Gharbi A. and Bouda A.; Energy spectra of Hartmann and ring-shaped oscillator potentials using the quantum Hamilton-Jacobi formalism, Phys. Scr. 88, 045007 (2013)

[9] Bharali A.; Systematic search of exactly solvable ring-shaped potential using the transformation method, Phys. Scr. 88, 035009 (2013)

[10] Dong-Sheng Sun, Yuan You, Fa-Lin Lu, Chang-Yuan Chen and Shi-Hai Dong; The quantum characteristics of a class of complicated double ring-shaped non-central potential, Phys. Scr. 89, $045002(2014)$

[11] Gribakin G.F. and Swann A.R., Effect of dipole polarizability on positron binding by strongly polar molecules, J. Phys. B 48, 215101 (2015)

[12] Fermi E. and Teller E., The Capture of Negative Mesotrons in Matter, Phys. Rev. 72, 399 (1947)

[13] Turner J.E.; Minimum dipole moment required to bind an electron-molecular theorists rediscover phenomenon mentioned in Fermi-Teller paper twenty years earlier, Am. J. Phys. 45, 758 (1977)

[14] Fox K. and Turner J.E.; Variational Calculation for Bound States in an Electric-Dipole Field, J. Chem. Phys. 45, 1142 (1966) 
[15] Crawford O. and Dalgarno A.; Bound states of an electron in a dipole field, Chem. Phys. Lett. 1, $23(1967)$

[16] Gutsev G.L. and Adamowicz L.; Electronic and geometrical structure of dipole-bound anions formed by polar molecules, J. Phys. Chem. 99, 13412-13421 (1995)

[17] Jordan K.D. and Wang F.; Theory of Dipole-Bound Anions, Annu. Rev. Phys. Chem. 54, 367-396 (2003)

[18] Svozil D., Jungwirth P. and Havlas Z.; Electron Binding to Nucleic Acid Bases. Experimental and Theoretical Studies. A Review, Collect. Czech. Chem. Commun. 69, 1395-1428 (2004)

[19] Simons J.; Molecular Anions, J. Phys. Chem. A 112, 6401-6511 (2008)

[20] Connolly K. and Griffiths D.J., Critical dipoles in one, two, and three dimensions, Am. J. Phys. 756 (2007)

[21] Glasser M.L. and Nieto L.M.; Electron capture by an electric dipole in two dimensions, Phys. Rev. A 75, 062109 (2007)

[22] AlHaidari A.D.; Analytic Solution of the Schrödinger Equation for an Electron in the Field of a Molecule with an Electric Dipole Moment, Ann. Phys. 3231709 (2008)

[23] Moumni M. and Falek M.; Schrödinger Equation for Non-Pure Dipole Potential in 2D Systems, J. Math. Phys. 57, 072104 (2016)

[24] Görlitz A; et al.; Realization of Bose-Einstein Condensates in Lower Dimensions, Phys. Rev. Lett. 87, 130402 (2001)

[25] Martiyanov K., Makhalov V. and Turlapov A.; Observation of a Two-Dimensional Fermi Gas of Atoms, Phys. Rev. Lett. 105, 030404 (2010)

[26] Safonov, A.I., Vasilyev S.A., Yasnikov I.S., Lukashevich I.I. and Jaakkola S.; Observation of Quasicondensate in Two-Dimensional Atomic Hydrogen, Phys. Rev. Lett. 81, 4545-4548 (1998)

[27] Zhou J.L. and Xiong J.J.; Hydrogen molecular ions in two dimensions, Phys. Rev. B 41, 12274-12277 (1990)

[28] Vasilyev S., Jarvinen J., Safonov A.I., Kharitonov A.A., Lukashevich I.I. and Jaakkola S.; Electron-Spin-Resonance Instability in Two-Dimensional Atomic Hydrogen Gas, Phys. Rev. Lett. 89, 153002 (2002)

[29] Gadella M., Negro J., Nieto L.M. and Pronko G.P.; Two Charged Particles in the Plane Under a Constant Perpendicular Magnetic Field, Int. J. Theor. Phys. 50, 2019-2028 (2011)

[30] De Martino A., Klöpfer D., Matrasulov D.U. and Egger R.; Electric-Dipole-Induced Universality for Dirac Fermions in Graphene, Phys. Rev. Lett. 112, 186603 (2014)

[31] Klöpfer D., De Martino A., Matrasulov D.U. and Egger R.; Scattering theory and ground-state energy of Dirac fermions in graphene with two Coulomb impurities, Eur. Phys. J. B 87, 187 (2014) 
[32] Gindikin Y. and Sablikov V. A.; Spin-orbit-driven electron pairing in two dimensions, Phys. Rev. B 98, 115137 (2018)

[33] Kratzer A.; Die ultraroten Rotationsspektren der Halogenwasserstoffe, Z. Phys. 3, 289-307 (1920)

[34] Kratzer A.; Die Gesetzmässigkeiten in den Bandspektren, Enc. d. Math. Wiss. 3, 821-859 (1926)

[35] Fortunato L. and Vitturi A.; Analytically solvable potentials for gamma unstable nuclei, J.Phys. G 29, 1341-1350 (2003)

[36] Hajigeorgiou P.G.; Exact analytical expressions for diatomic rotational and centrifugal distortion constants for a Kratzer Fues oscillator, J. Molec. Spect. 235, 111-116 (2006)

[37] Berkdemir C., Berkdemir A. and Han J.; Bound state solutions of the Schrödinger equation for modified Kratzer's molecular potential, Chem. Phys. Lett. 417, 326-329 (2006)

[38] Van Hooydonk G.; Ionic Kratzer bond theory and vibrational levels for achiral covalent bond $H H$, Z. Naturforsch. A 64, 801 (2009)

[39] Batra K. and Prasad V.; Spherical quantum dot in Kratzer confining potential: study of linear and nonlinear optical absorption coefficients and refractive index change, Eur. Phys. J. B 91, 298 (2018)

[40] Cheng Y.F. and Dai T.Q.; Exact solution of the Schrödinger equation for the modified Kratzer potential plus a ring-shaped potential by the Nikiforov-Uvarov method, Phys. Scr. 75, 274-277 (2007)

[41] Babaei-Brojeny A.A. and Mokari M.; An analysis of the applications of the modified Kratzer potential, Phys. Scr. 84045003 (2011)

[42] Molas M.R., Slobodeniuk A.O., Nogajewski K., Bartos M., Bala L., Babinski A., Watanabe K., Taniguchi T., Faugeras C. and Potemski M.; Energy spectrum of two-dimensional excitons in a non-uniform dielectric medium, arXiv:1902.03962

[43] Sivoukhine D.; Cours de physique générale - Tome 5. Physique atomique et nucléaire - Première partie; French Traduction Editions MIR pp 223 (1986) ASIN: B00AW98XK8

[44] Molski M. and Konarski J.; Modified kratzer-fues formula for rotation-vibration energy of diatomic molecules, Acta. Phys. Pol. A 82, 927-936 (1992)

[45] Durmus A.; Non-relativistic treatment of diatomic molecules interacting with a generalized Kratzer potential in hyperspherical coordinates, J. Phys. A: Math. Theor. 44, 155205 (2011)

[46] Bao J. and ShizgalB.D.; Pseudospectral method of solution of the Schrödinger equation for the Kratzer and pseudoharmonic potentials with nonclassical polynomials and applications to realistic diatom potentials, Comp. Theor. Chem. 1149, 49-56 (2019)

[47] Mathieu E., Mémoire sur le mouvement vibratoire d'une membrane de forme elliptique, J. Math. Pures. Appl. 13, 137 (1868) 
[48] Abramowitz M. and Stegun I.A., Handbook of Mathematical Functions, Dover Publ., New York, (1972)

[49] Floquet G., Sur les équations différentielles linéaires à coefficients périodiques, Annales de l'École Normale Supérieure 1247 (1883)

[50] Bloch F., Über die Quantenmechanik der Elektronen in Kristallgittern, Z. Physik 52555 (1928)

[51] https://dlmf.nist.gov/28.6 (2018-12-15)

[52] Saad N. and Hall R.L.; Integrals containing confluent hypergeometric functions with applications to perturbed singular potentials, J. Phys. A: Math. Gen. 36, 7771-7788 (2003)

[53] Oyewumi K.J.; Analytical Solutions of the Kratzer-Fues Potential in an Arbitrary Number of Dimensions, Found. Phys. Lett. 18, 75 (2005)

[54] Agboola A.; Complete Analytic Solutions of the Mie-type Potentials in N-Dimensions, Acta. Phys. Polonica A 120, 371 (2011)

[55] Zaslow B. and Zandler M.E., Two-Dimensional Analog to the Hydrogen Atom, Am. J. Phys. 35, $1118(1967)$

[56] Parfitt D.G.W. and Portnoi M.E., The two-dimensional hydrogen atom revisited, J. Math. Phys. 43, 4681 (2002)

[57] Hecht K.T. and Adler A.; Generalized seniority for favored $J \neq 0$ pairs in mixed configurations, Nucl. Phys. A. 137 129-143 (1969)

[58] Arima A., Harvey M. and Shimizu K.; Pseudo LS coupling and pseudo SU(3) couling shemes, Phys. Lett. B. 30 517-522 (1969)

[59] Ginocchio J.N.; Pseudospin as a Relativistic Symmetry, Phys. Rev. Lett. 78, 436 (1997)

[60] Zhou S.G., Meng J. and Ring P.; Spin Symmetry in the Antinucleon Spectrum, Phys. Rev. Lett. 91, 262501 (2003)

[61] Liang H., Meng J. and Zhou S.G.; Hidden pseudospin and spin symmetries and their origins in atomic nuclei, Phys. Rep. 570 1-84 (2015)

[62] Hall R.L. and Yeşiltaş Ö.; Comparison theorems for the Dirac equation with spin-symmetric and pseudo-spin-symmetric interactions, J. Phys. A Math. Theor. 43, 195303 (2010)

[63] Berkdemir C. and Sever R.; Pseudospin symmetry solution of the Dirac equation with an angle-dependent potential, J. Phys. A Math. Theor.41, 045302 (2008)

[64] Pauli W. Jr.; Zur Quantenmechanik des magnetischen Elektrons, Z. Phys. 43, 601 (1927)

[65] Snyder H. and WeinbergJ., Stationary States of Scalar and Vector Fields, Phys. Rev. 57, 307 (1940)

[66] Snyder H. and WeinbergJ., On The Existence of Stationary States of the Mesotron Field, Phys. Rev. 57, 315 (1940) 
[67] Schulze-Halberg A. and Roy P., Bound states of the two-dimensional Dirac equation for an energy-dependent hyperbolic Scarf potential , J. Math. Phys. 58, 113507 (2017)

[68] Schulze-Halberg A. and Yeşiltaş Ö.; Generalized Schrödinger equations with energy-dependent potentials: Formalism and applications, J.Math.Phys. 59, 113503 (2018)

[69] Benzair, H. Merad and M. Boudjedaa, T.; Electron propagator with vector and scalar energydependent potentials in (2+1)-dimensional space-time, Int. J. Mod. Phys. A 331850186 (2018)

[70] Gradshteyn I.S. and Ryzhik I.M., Table of Integrals, Series, and Products, Alan Jeffrey and Daniel Zwillinger (eds.) Elsevier, London (2007)

[71] Saad N., Hall R.L. and Ciftci H.; The Klein-Gordon equation with the Kratzer potential in d dimensions, Cent. Eur. J. Phys. 6, 717-729 (2008)

[72] Berkdemir C., Relativistic treatment of a spin-zero particle subject to a Kratzer-type potential, Am. J. Phys. 75, 81 (2007)

[73] Alhaidari A.D., Bahlouli H. and Al-Hasan A.; Dirac and Klein Gordon equations with equal scalar and vector potentials, Phys. Lett. A 349, 87-97 (2006)

[74] Durmus A. and Yasuk F.; Relativistic and nonrelativistic solutions for diatomic molecules in the presence of double ring-shaped Kratzer potential, J. Chem. Phys. 126, 074108 (2007)

[75] Ikhdair S.M. and Sever R.; Relativistic solution in D-dimensions to a spin-zero particle for equal scalar and vector ring-shaped potential, Cent. Eur. J. Phys. 6, 141-152 (2008) 